Recepción: 01 / 03/ 2018

Aceptación: 15 / 05 / 2018

Publicación: 07 / 07 / 2018

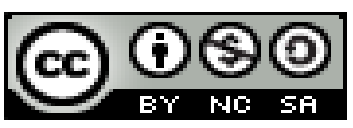

Ciencias Agrícolas

Artículo de Investigación

\title{
Efecto de caracol manzana (Pomacea canaliculata) en plántulas de arroz en piscina con y sin lámina de agua
}

Effect of apple snail (Pomacea canaliculata) on rice seedlings in a pool with and without a sheet of wáter

Efeito do caracol de maçã (Pomacea canaliculata) em mudas de arroz em piscina com e sem lençol freático

Erik B. Vargas-Hernández I

erik.vargas@hotmail.com

Ángel P. Jines-Carrasco II

angel.jinesc@ug.edu.ec

Víctor H. Verdezoto-Vargas ${ }^{\text {III }}$

victor.verdezotov@ug.edu.ec

Gonzalo I. Almagro-Mayorga VI

gonzalo.almagrom@ug.edu.ec

Iván E. Ramos-Mosquera V

ivan.ramosm@ug.edu.ec

Correspondencia: erik.vargas@hotmail.com

I. Ingeniero Agrónomo de la Universidad de Guayaquil, Guayaquil, Ecuador.

II. Docente de la Facultad de Ciencias Agrarias de la Universidad de Guayaquil, Guayaquil, Ecuador.

III. Docente de la Facultad de Ciencias Agrarias de la Universidad de Guayaquil, Guayaquil, Ecuador.

IV. Docente de la Facultad de Ciencias Agrarias de la Universidad de Guayaquil, Guayaquil, Ecuador.

v. Docente de la Facultad de Ciencias Agrarias de la Universidad de Guayaquil, Guayaquil, Ecuador. 
Erik B. Vargas-Hernández; Ángel P. Jines-Carrasco; Víctor H. Verdezoto-Vargas; Gonzalo I. Almagro-Mayorga; Iván

\section{Resumen}

El caracol manzana (Pomacea canaliculata) causa grandes daños en el cultivo de arroz, ataca en su primera fase de crecimiento de las plántulas. La presente investigación se realizó en el recinto Zapan, cantón Samborondon, provincia del Guayas, el objetivo fue evaluar los efectos que causa el caracol manzana a las plántulas de arroz en diferentes días de edad, en piscina con y sin lámina de agua. Se utilizó un diseño de bloques completamente al azar con tres repeticiones, colocando 5 caracoles $/ \mathrm{m}^{2}$ por tratamiento, transplantadas en edades de 20, 25, 30 días. Se evaluaron el número de plántulas devoradas a las 24, 48, 72 y 96 horas después del trasplante, siendo más susceptible las plántulas con 25 días de edad (T3), bajo lámina de agua de $5 \mathrm{~mm}$, presento un promedio de 176,33 de plántulas devoradas a las 24 horas, en la misma edad de las plántulas en piscina sin agua fue mayor con un promedio de 65,67 plantas devoradas.

Palabras claves: Caracol manzana, daños, plántulas, arroz, lámina de agua. 


\section{Abstract}

The apple snail (Pomacea canaliculata) causes great damage to the rice crop, it attacks in its first phase of seedling growth. The present investigation was carried out in the Zapan enclosure, canton Samborondon, province of Guayas, the objective was to evaluate the effects that the apple snail causes on rice seedlings in different days of age, in pools with and without water sheet. A completely randomized block design with three repetitions was used, placing 5 snails / $\mathrm{m} 2$ per treatment, transplanted at ages of 20, 25, 30 days. The number of seedlings devoured at 24, 48, 72 and 96 hours after the transplant was evaluated, being more susceptible the seedlings with 25 days of age (T3), under sheet of water of $5 \mathrm{~mm}$, I present an average of 176.33 of seedlings devoured at 24 hours, in the same age of the seedlings in pool without water was higher with an average of 65.67 plants devoured.

Key words: Snail apple, damage, seedlings, rice, sheet of water. 
Erik B. Vargas-Hernández; Ángel P. Jines-Carrasco; Víctor H. Verdezoto-Vargas; Gonzalo I. Almagro-Mayorga; Iván

\section{Introducción.}

El arroz es considerado como un alimento básico para la mitad de la población en el mundo, convirtiéndose en un producto agrícola importante, generando ingresos a lo largo del siglo XX, el mismo ha ido evolucionando desde un cultivo de secano, hasta convertirse en un cultivo altamente tecnificado y productivo, cultivado en las últimas décadas, el sistema de riego (Sanint, 2010).

La producción Mundial de Arroz en el 2016, según el informe de seguimiento del Mercado de la FAO (2017), debería de totalizar 751,9 millones de toneladas, siendo 3,9 millones de toneladas más que las previsiones de diciembre y 1,6 por ciento por encima del bajo nivel de 2015, y estima que la cifra de producción para el año 2017 será de 758,9 millones de toneladas, implicando un crecimiento del 0,9 por ciento anual.

Según datos registrados por la ESPAC y el INEC durante los últimos seis años se ha ubicado como el segundo producto con mayor superficie cosechada después del cacao, y el cuarto producto con mayor producción tras la caña de azúcar, el banano, y la palma africana (Moreno Aguirre \& Salvador Sarauz, 2014).

Según la estimación de superficie sembrada de arroz del 2016 se registró 364,112 hectáreas. En el primer cuatrimestre Guayas tiene el 59\%, Los Ríos 36\%, Manabí 2\%, y en las provincias de Loja y El Oro aportan con el 1\% cada una de ellas. En el segundo cuatrimestre sembraron 196,226 distribuidas en Guayas 72\%, Los Ríos 24\%, Manabí 3\% y en las provincias de Loja y El Oro 1\% entre las dos. El tercer cuatrimestre hubo 29,802 hectáreas, donde Guayas aporta 80\%, en Los Ríos 17\%, y en Manabí 3\% (Sinagap, 2016a). 
El rendimiento nacional del cultivo de arroz en cáscara para el tercer cuatrimestre del año 2016 fue de 6.81 toneladas por hectárea, donde la provincia con el mejor rendimiento fue Loja con 9.61 toneladas por hectárea, y la provincia del Guayas obtuvo un rendimiento de 6.8 toneladas por hectárea, siendo cantones: Santa Lucía, Daule y Colimes con los mejores rendimientos, por otra parte los cantones de menor se encuentran; Yaguachi, Baba y Alfredo Baquerizo Moreno, según los productores esta baja esta relacionan con los problemas fitosanitarios (Sinagap, 2016b).

En Ecuador el caracol manzana (Pomacea canaliculata) es una plaga invasora introducida, reportada en el año 2005 en el recinto San Mauricio del cantón El Triunfo, causando grandes daños en el cultivo de arroz, la misma que ataca en su primera fase de crecimiento de las plántulas y pone en peligro su rentabilidad, afectan directamente el costo de producción en un $40 \%$ a causa de altas poblaciones, y se ha convertido en una amenaza en todas las áreas arroceras del Litoral Ecuatoriano (Villacis Gavilanez, 2014).

Arias, (2011) dice que la solución para controlar la plaga del caracol manzana, más no para erradicar es casi imposible, por lo que se recomienda es realizar prácticas culturales: como recolectar los huevecillos, siembra tardía de trasplante, no es recomendable para el manejo de esta plaga la siembra directa, y la nivelación de los suelos es de mucha importancia, al ser este molusco acuático se concentra en los charcos.

El objetivo de la presente investigación es evaluar los efectos que causa el caracol manzana a las plántulas de arroz en diferentes días de edad, en piscina con y sin lámina de agua. 
Erik B. Vargas-Hernández; Ángel P. Jines-Carrasco; Víctor H. Verdezoto-Vargas; Gonzalo I. Almagro-Mayorga; Iván E. Ramos-Mosquera

\section{Metodología.}

El presente trabajo de investigación se realizó en el recinto Zapan, cantón Samborondon, provincia del Guayas. Se encuentra ubicado entre las coordenadas UTM 9782245,9 norte y 636348,7 sur.

\section{CLIMA}

Altura

Temperatura promedio

anual

Humedad relativa

Precipitación media

anual

Vientos

Heliofania

Fisiografía

Suelos

\section{CÁLIDO HÚMEDO}

$3.5 \mathrm{msnm}$

$24^{\circ} \mathrm{C}-25^{\circ} \mathrm{C}$

Semi-humeda 70\%

$1000 \mathrm{~mm}-2000 \mathrm{~mm}$

$$
3.4 \mathrm{~m} / \mathrm{s}
$$

1593 horas luz/año

Pendiente $2 \%$

Franco arcilloso

Fuente: (Gobierno Autónomo Descentralizado Parroquil Rural de Tarifa, 2015)

\section{Material biológico}

- $\quad$ variedad INIAP-14

- $\quad$ especímenes de caracol ( Pomácea canaliculata).

\section{Maquinaria}

- motocultor de fangueo 
- $\quad$ bomba de motor.

Herramientas y equipos

- $\quad$ machete, espeque, cinta métrica, tableros

- $\quad$ bomba de mochila, cámara fotográfica

- $\quad$ libreta de campo

\section{Metodología}

Diseño de la investigación

a) Factores estudiados

\section{Factor 1. Plantas de diferentes días de edad}

[1] E1.20 días de edad (dde)

[2] E2. 25 días de edad (dde)

[3] E3. 30 días de edad (dde)

\section{Factor 2. Piscina con y sin agua}

- $\quad$ P1. Piscina con agua (lámina de $5 \mathrm{~cm}$ )

- $\quad$ P2. Piscina sin agua

b) Tratamientos en estudio 
Erik B. Vargas-Hernández; Ángel P. Jines-Carrasco; Víctor H. Verdezoto-Vargas; Gonzalo I. Almagro-Mayorga; Iván E. Ramos-Mosquera

\section{Cuadro ${ }^{\circ} 1$ Tratamientos estudiados}

$\mathrm{N}^{\circ}$. Tratamientos Código

$1 \quad$ A1B1 Plantas de 20 días de edad en piscina con agua

2 A1B2 Plantas de 20 días de edad en piscina sin agua

3 A2B1 Plantas de 25 días de edad en piscina con agua

$4 \quad$ A2B2 Plantas de 25 días de edad en piscina sin agua

$5 \quad$ A3B1 Plantas de 30 días de edad en piscina con agua

6 A3B2 Plantas de 30 días de edad en piscina sin agua

c) Diseño experimental

Se utilizó un diseño de bloques completamente al azar (DBCA) con arreglo factorial $3 \times 2$, con tres (3) repeticiones.

Para el análisis estadístico se realizó el análisis de varianza de la prueba de Duncan al 0,05\% y $0,01 \%$ de probabilidades para determinar la diferencia entre las medias de los tratamientos y se delimito cual fue menos susceptible al ataque del caracol.

A continuación, se expresan las fuentes de variación y grados de libertad que se utilizaron para el Análisis de varianza. 
Cuadro ${ }^{\circ} 2$ Fuentes de variación y grados de libertad para el Análisis de varianza

Fuentes de variación Grados de libertad

Tratamiento (t-1) 5

Repeticiones (r-1) 2

Edad de trasplante 2

Lámina de agua 1

Error experimental (t-1)(r-1) 10

Total (t x r)-1 17

c) Unidad experimental

La unidad experimental fue una parcela para tomar la información, en la que se evaluó el número de plantas comidas por caracol.

d) Delineamiento experimental

Ancho de parcela $2 \mathrm{~m}$

Largo de parcela $3 \mathrm{~m}$

Área de parcela $\quad 6 \mathrm{~m} 2$

Distancia entre bloque $\quad 1.5 \mathrm{~m}$

Distancia entre parcela $\quad 1 \mathrm{~m}$ 
Erik B. Vargas-Hernández; Ángel P. Jines-Carrasco; Víctor H. Verdezoto-Vargas; Gonzalo I. Almagro-Mayorga; Iván

Área útil $108 \mathrm{~m} 2$

Área total del ensayo $204 \mathrm{~m} 2$

e) Manejo del cultivo

Preparación de suelo

Se realizó el pase del motocultor (fangueo), con ayuda de una tabla se nivelo el terreno, y se procedió hacer la respectiva delimitación de las parcelas.

Pre-germinación

Se colocó la semilla en un balde a remojar durante 24 horas, después se sacó del agua luego de transcurrir 24 horas se empezó a sembrar en el semillero.

\section{Construcción del semillero}

Se realizó un semillero en un área de 2 por 3 metros.

\section{Control de malezas}

El control de malezas se realizó una aplicación de herbicidas post-emergente después del transplante y la demás se realizó manual.

Riego

Se realizó con un motor-bomba para llenar las piscinas a cinco (5) centímetros. 


\section{Fertilización}

Se realizó la aplicación a los 30 días del transplante del cultivo.

\section{Controles fitosanitarios}

Se realizó monitoreos en todas las fases de desarrollo del cultivo (inicio - vegetativa reproductiva - maduración), manteniendo el umbral económico y el porcentaje de daño no paso el umbral.

\section{Cosecha}

Se realizó en forma manual cuando las plantas alcanzaron su madurez fisiológica y el $95 \%$ de los granos en las panículas presentaron color pajizo y que la espiga se encontraba totalmente doblada.

\section{f) Manejo del experimento}

Para el inicio del experimento se recolecto los caracoles en el campo y se colocó 5 caracoles por metro cuadrado por tratamiento y repetición.

El trasplante se realizó con tres edades diferentes (20, 25, 30 días) en piscina con agua y sin agua. El nivel de agua para piscina con agua fue de cinco centímetros. Cada parcela se sembró a una distancia de 0,25 x 0,25 metros y cinco plántulas por sitio por parcela se sembraron 6930 plantas. Se prepararon parrillas de 2 x 3 metros (6m”) se nivelaron, se realizaron riegos constante para mantener la lámina de agua y se dejaron en permanente. 
Erik B. Vargas-Hernández; Ángel P. Jines-Carrasco; Víctor H. Verdezoto-Vargas; Gonzalo I. Almagro-Mayorga; Iván E. Ramos-Mosquera

\section{Resultados.}

En el cuadro $\mathrm{N}^{\circ}$ 3. Se presentan los promedios de las plantas devoradas por caracol manzana ( Pomacea Canaliculata) en la piscina con agua:

- A las 24 horas el tratamiento que presento mayor número de plántulas devoradas fue el T3 (plantas 25 días de edad) con un promedio de 176,33, y el menor fue en plantas de 20 días de edad (T1), (Figura 1)

- A las 48 horas el T5 (plantas 30 días de edad) fue mayor, con un promedio de 55 plántulas comidas, (Figura 2).

Los datos no presentan diferencia estadística entre sí, pero si diferencias numéricas.

Cuadro $N^{\circ} 1$ Promedios de plantas devoradas por caracol en piscina con agua. Tarifa.

Samborondon, 2017.

\begin{tabular}{|c|c|c|c|c|}
\hline \multicolumn{5}{|c|}{ PLANTAS DEVORADAS POR CARACOL EN PISCINA CON AGUA } \\
\hline TRATAMIENTOS & 24 HORAS & 48 HORAS & 72 HORAS & 96 HORAS \\
\hline $\begin{array}{c}\text { T1 } \\
\text { PLANTAS 20 } \\
\text { DIAS DE EDAD }\end{array}$ & $63,33 \mathrm{~A}$ & $24,33 \mathrm{~A}$ & $56,33 \mathrm{~A}$ & $25,67 \mathrm{~A}$ \\
\hline $\begin{array}{c}\text { T3 } \\
\text { PLANTAS 25 } \\
\text { DIAS DE EDAD }\end{array}$ & $176,33 \mathrm{~A}$ & $53,33 \mathrm{~A}$ & $50,33 \mathrm{~A}$ & $56,00 \mathrm{~A}$ \\
\hline $\begin{array}{c}\text { T5 } \\
\text { PLANTAS 30 } \\
\text { DIAS DE EDAD }\end{array}$ & $113,00 \mathrm{~A}$ & $55,00 \mathrm{~A}$ & $42,67 \mathrm{~A}$ & $10,33 \mathrm{~A}$ \\
\hline $\mathrm{CV}$ & $21.75 \%$ & $17.25 \%$ & $17.73 \%$ & $47.32 \%$ \\
\hline Ftabla & NS & NS & NS & NS \\
\hline
\end{tabular}

Valores con la misma letra no tiene diferencia estadística basados en la prueba de Duncan al $0.05 \%$ 
Piscina sin agua

Los promedios de las plantas devoradas por caracol (Pomacea canaliculata) en la piscina sin agua, los datos no presentan diferencia estadística entre sí, pero si diferencias numéricas, el tratamiento que presento mayor fue el T4 con un promedio de 65,67 plantas devoradas a las 24 horas.

Cuadro $N^{\circ}$ 2.- Promedios de plantas devoradas por caracol en piscina sin agua. Tarifa.

Samborondon, 2017

\begin{tabular}{|c|c|c|c|c|}
\hline \multicolumn{5}{|c|}{ PLANTAS DEVORADAS POR CARACOL EN PISCINA SIN AGUA } \\
\hline TRATAMIENTOS & 24 HORAS & 48 HORAS & 72 HORAS & 96 HORAS \\
\hline $\begin{array}{c}\text { T2 } \\
\text { PLANTAS 20 } \\
\text { DÍAS DE EDAD }\end{array}$ & 29,00 a & 19,67 a & 39,00 & 32,00 \\
\hline $\begin{array}{c}\text { T4 } \\
\text { PLANTAS 25 } \\
\text { DÍAS DE EDAD }\end{array}$ & 65,67 a & 25,33 a & 20,00 & 11,00 \\
\hline $\begin{array}{c}\text { T6 } \\
\text { PLANTAS 30 } \\
\text { DÍAS DE EDAD }\end{array}$ & 19,67 a & 8,33 a & 5,67 & 2,67 \\
\hline CV & $28.72 \%$ & $20.10 \%$ & $27.90 \%$ & $37.30 \%$ \\
\hline Ftabla & NS & NS & $*$ & $*$ \\
\hline
\end{tabular}

Valores con la misma letra no tiene diferencia estadística basados en la prueba de Duncan al $0.05 \%$

Estimado de pérdidas de plántulas de arroz ocasionados por daños de caracol

Se estima que en una hectárea se siembra aproximadamente 601666 plántulas de arroz a razón de cinco plantas por sitio. La producción estimada es de 50 sacas por hectárea de 200 libras. Precio estimado por saca $\$ 28,00$ dólares. 
Erik B. Vargas-Hernández; Ángel P. Jines-Carrasco; Víctor H. Verdezoto-Vargas; Gonzalo I. Almagro-Mayorga; Iván E. Ramos-Mosquera

Cuadro $N^{\circ}$ 3.- Estimado de pérdidas de plántulas de arroz ocasionados por daños de caracol durante cuatro días (96 horas) en piscina con agua, Tarifa, Samborondon, 2017

\begin{tabular}{|c|c|c|c|c|c|c|c|}
\hline $\begin{array}{c}\text { Plántulas/ } \\
\text { Transpl/ } \\
\text { días }\end{array}$ & $\begin{array}{c}\text { Número } \\
\text { plántulas } \\
\text { comidas }\end{array}$ & $\begin{array}{c}\text { Pérdida } \\
\text { s en } \\
\text { sacas }\end{array}$ & $\begin{array}{c}\% \\
\text { pérdid } \\
\text { a }\end{array}$ & $\begin{array}{c}\text { Pérdida } \\
\text { total en } \\
\text { dólares }\end{array}$ & $\begin{array}{c}\text { Producci } \\
\text { ón } \\
\text { /ha } \\
\text { SACAS }\end{array}$ & $\begin{array}{c}\text { Venta } \\
\text { estimada de } \\
\text { la producción }\end{array}$ & $\begin{array}{c}\text { Utilidad } \\
\text { neta por } \\
\text { tratamiento }\end{array}$ \\
\hline 20 & 282777 & 23.49 & 46.99 & 657.98 & 50 & 1400 & 742.01 \\
\hline 25 & 560000 & 46.53 & 93.07 & 1303.05 & 50 & 1400 & 96.95 \\
\hline 30 & 368333 & 30.61 & 61.22 & 857.07 & 50 & 1400 & 542.94 \\
\hline
\end{tabular}

Cuadro $N^{\circ}$ 4.- Estimado de pérdidas de plántulas de arroz ocasionados por daños de caracol durante cuatro días (96 horas) en piscina sin agua, Tarifa, Samborondon, 2017

\begin{tabular}{|c|c|c|c|c|c|c|c|}
\hline $\begin{array}{c}\text { Plántulas/ } \\
\text { Transpl/ } \\
\text { días }\end{array}$ & $\begin{array}{c}\text { Número } \\
\text { plántulas } \\
\text { comidas }\end{array}$ & $\begin{array}{c}\text { Pérdidas } \\
\text { en } \\
\text { sacas }\end{array}$ & $\begin{array}{c}\% \\
\text { pérdida }\end{array}$ & $\begin{array}{c}\text { Pérdida } \\
\text { total en } \\
\text { dólares }\end{array}$ & $\begin{array}{c}\text { Venta } \\
\text { Producción } \\
\text { /ha } \\
\text { SACAS }\end{array}$ & $\begin{array}{c}\text { estimada } \\
\text { de la } \\
\text { producción }\end{array}$ & $\begin{array}{c}\text { Utilidad } \\
\text { neta por } \\
\text { tratamiento }\end{array}$ \\
\hline 20 & 199444 & 16.57 & 33.15 & 464.08 & 50 & 1400 & 935.92 \\
\hline 25 & 203333 & 16.90 & 33.80 & 473.13 & 50 & 1400 & 926.87 \\
\hline 30 & 60555 & 5.03 & 10.06 & 140.90 & 50 & 1400 & 1259.10 \\
\hline
\end{tabular}

Se estima que en una hectárea se siembra aproximadamente 601666 plántulas de arroz a razón de cinco plantas por sitio. La producción estimada es de 50 sacas por hectárea de 200 libras. Precio estimado por saca $\$ 28,00$ dólares.

\section{Discusión.}

Los daños que produce este molusco en el cultivo depende de la edad y el tiempo que permanece en situaciones favorables, siendo más susceptible las plántulas de 25 días de edad, bajo lámina de agua de $5 \mathrm{~cm}$ a una densidad de cinco caracoles por metro cuadrado, causaron un $45 \%$ 
de daño a las primeras 24 horas después del transplante, con especímenes de $1.2 \mathrm{~cm}$ a $1.5 \mathrm{~cm}$ de diámetro, teniendo un apetito voraz, siendo estos datos menores a trabajo realizado por Ochoa y García (2012) donde dice que la densidad poblacional y edad de 3 caracoles por metro cuadrado causa pérdidas significativas en plántulas de hasta 18-21 días cuando la siembra es por trasplante, teniendo diámetros mayores de una densidad los individuos de $4 \mathrm{~cm}$ son más destructivos, que pueden llegar al $20 \%$ de destrucción.

Los especímenes con el diámetro de $1.2 \mathrm{~cm}-1.5 \mathrm{~cm}$ presentan un apetito considerable donde el promedio de plántulas comida por día fue de 1.67 - 5.87, donde las situaciones ambientales para los caracoles eran favorables con una lámina de $5 \mathrm{~mm}$, mientras la voracidad fue menor en condiciones desfavorables que estuvo solo a capacidad de campo o suelos saturados fue $0.36-2.18$ plántulas por día estos datos concuerdan con Ochoa Chumaña \& Garcia Onofre , (2012) donde señala que dependiendo el tamaño de la concha se relaciona con el número de plantas comidas, los caracoles con una altura de $1 \mathrm{~cm}$ son muy pequeños para alimentarse de las plántulas.

Los caracoles (Pomacea canaliculata ) comienzan alimentandose desde la parte superior del nudo la que divide la raíces adventicias con el tallo herbáceos de la plántula de arroz, donde la edad de la planta es más susceptibles, se vuelve vulnerable por el stress que pasa por la adaptación del transplante, son más activos por las tarde cuando baja de temperatura, estos resultados son similares a Ochoa y García (2012), que definen que el daño causado por los caracoles P. canaliculata cortan la base de las plántulas con su rádula y devoran tallos y las hojas más tiernas y suculentas, prefieren el tejido suave de la planta. Los caracoles son más activos en el ataque a las plantas de arroz durante la noche y al amanecer. 
Erik B. Vargas-Hernández; Ángel P. Jines-Carrasco; Víctor H. Verdezoto-Vargas; Gonzalo I. Almagro-Mayorga; Iván

\section{Conclusiones.}

Terminada la investigación sobre la incidencia de los caracoles en la mortalidad de plantas de diferentes días de edad se llegó a las siguientes conclusiones:

El caracol manzana (Pomácea canaliculata) prefieren plántulas tiernas y susceptibles y cuando la lámina de agua les permita tener las condiciones apropiadas y donde su apetito es más voraz para realizar el daño las plantas de arroz.

La edad de la plántula más susceptible al ataque del caracol manzana fue la de 25 días en piscina con lámina de agua que llego a tener en un día el 54.90\% de plántulas devoradas, mientras que en las primeras 24 horas la voracidad de Pomacea canaliculata fue de $45.79 \%$, luego en las 48 horas tuvo una mortalidad del $30.74 \%$, a las 72 horas hubo un incremento de plantas comidas del $37.36 \%$, posteriormente a las 96 horas llego hacer un daño de $54.90 \%$.

Las condiciones menos favorables para el caracol manzana (Pomacea canaliculata) son piscina sin lámina de agua con suelo solo saturados y con plántulas de edad de 30 días donde el promedio de voracidad por día es de $5.10 \%$ a las 24 horas, en 48 horas baja su voracidad al 2.36\%, posteriormente a las 96 horas llego hacer un daño de plántulas comidas de $0.79 \%$.

Los caracoles de un diámetro de $1.2 \mathrm{~cm}$ a $1.5 \mathrm{~cm}$ en estado juvenil, pueden llegar a alimentarse hasta un promedio de 5,87 plántulas/día, en situaciones favorables, mientras que en estado desfavorables o con suelos saturado o en lodo llegan a devorar 2.18 plántulas por día. 
Los daños causados por el caracol manzanas son principalmente en el tallo (la parte superior al nudo) donde es más tierno y susceptible el tejido de las plántulas para el ataque, este molusco prefiere alimentarse a partir de la puesta del sol cuando baja la temperatura.

\section{Bibliografia.}

Gobierno Autónomo Descentralizado Parroquil Rural de Tarifa. (2015). Recuperado el 07 de 07 de 2017 , de http://app.sni.gob.ec/snilink/sni/PORTAL_SNI/data_sigad_plus/sigadplusdiagnostico/0968563930001_FASE\%20DI AGN\%C3\%93STICO\%20DEL\%20PLAN\%20DE\%20DESARROLLO\%20Y\%20ORDENA MIENTO\%20TERRITORIAL\%20PARROQUIAL\%20RURAL\%20DE\%20TARIFA\%2028 \%2010\%202015_30-10-2015_23-09-1

Moreno Aguirre , B., \& Salvador Sarauz , S. (2014). RENDIMIENTOS DEL ARROZ EN EL ECUADOR SEGUNDO CUATRIMESTRE DEL 2014. INIAP: Quito.

Sanint, L. R. (2010). Nuevos retos y grandes oportunidades tecnologicas para los sistemas arroceros: Produccion, seguridad alimentaria y disminucion de la pobreza en America Latina y el Caribe. En Produccion EcoEficiente del Arroz en América Latina (pág. 5). Cali, Colombia: Publicacion CIAT N 370.

Sinagap. (2016a). ESTIMACIÓN DE SUPERFICIE SEMBRADA DE ARROZ (Oryza sativa L.),MAÍZ AMARILLO DURO (Zea mays L.) Y SOYA (Glycine max) DEL AÑO 2016; EN LAS PROVINCIAS DE MANABÍ, LOS RÍOS, GUAYAS, SANTA ELENA, LOJA Y EL ORO. Obtenido de http://sinagap.agricultura.gob.ec/pdf/estudios_agroeconomicos/estimacion_superficie_arroz_ 2016.pdf

Villacis Gavilanez, J. A. (2014). CONTROL CULTURAL DEL CARACOL MANZANA (Pomacea canaliculata) EN EL CULTIVO DE ARROZ BAJO RIEGO EN EL SECTOR DE CEDEGE, CANTÒN BABAHOYO. Facultad de Ciencias Agropecuarias. Babahoyo: Universidad de Babahoyo. 